УДК 517.988 .8

\title{
О вычислении первых собственных чисел дискретного оператора с ядерной резольвентой с помощью метода Галеркина
}

\author{
Е. М. Малеко ${ }^{1}$
}

Аннотация: В статье рассматривается подход, позволяющий приближенно вычислять первые собственные числа дискретного оператора $A$ с ядерной резольвентой. Основная идея состоит в том, что для приближенного вычисления собственных чисел этого оператора его резольвента может быть заменена конечномерным матричным оператором. Собственные числа этого оператора, полученного методом Галеркина, являются приближенными собственными числами резольвенты. Обратные значения к найденным числам - приближенные собственные числа исходного дискретного оператора $A$. В конце статьи приведены два примера, в которых найдены достаточно точно первые собственные числа дискретных операторов с ядерной резольвентой двумя способоми: методом следов резолъвент, вычисленных точно, и с использованием метода Галеркина.

Ключевые слова: Собственные числа, дискретный оператор, ядерная резольвента.

Введение. В работе доказана сходимость собственных чисел матриц $\mathfrak{R}_{n}(A)$ к первым собственным числам ядерной резольвенты дискретного оператора $A$. "Приближающие"резольвенту оператора $A$ матрицы $\mathfrak{R}_{n}(A)$ будем строить по методу Галеркина. Покажем на конкретных примерах, что можно ограничиться лишь матричным представлением ядерной резольвенты дискретного оператора, чтобы при достаточных больших размерностях $n$ приближающих матриц с хорошей точностью получать первые собственные значения этого оператора.

1. Сходимость собственных чисел приближающих матриц $\mathfrak{R}_{n}(A)$ к первым собственным числам дискретного оператора $A$. Пусть $A$ - дискретный оператор, действующий в СГП $\mathbb{H}:=L_{2}(a, b)$ и имеющий там ядерную резольвенту; $\left\{\lambda_{i}\right\}_{i=1}^{\infty}-$ набор ненулевых собственных чисел оператора $A$, занумерованных по возрастанию модулей с учетом алгебраической кратности.

\footnotetext{
1 emaleko@rambler.ru
} 
Пусть дан матричный оператор

$$
\mathfrak{R}_{n}(A)=\left(b_{i j}\right)_{i, j=1}^{n}, \quad b_{i j}=\left(R_{0}(A) \varphi_{i}, \varphi_{j}\right),
$$

где $R_{0}(A)=A^{-1}$ - обратный оператор к $A,(\cdot, \cdot)$ - скалярное произведение в $\mathbb{H},\left\{\varphi_{i}\right\}_{i=1}^{\infty}-$ ортонормированный базис $(\mathrm{OHБ)} \mathrm{в} \mathbb{H}$. Хорошо известно, что для любой функции $\varphi \in \mathbb{H}$ функция $R_{0}(A) \varphi \in \mathcal{D}(A) \subset \mathbb{H}$. Матричным представлением определенного и ограниченного на всем $\mathbb{H}$ оператора $R_{0}(A)$ является, очевидно, оператор

$$
\mathfrak{R}_{\infty}(A)=\left(b_{i j}\right)_{i, j=1}^{\infty},
$$

а спектральными следами $g_{k}$ к-й степени оператора $R_{0}(A)-$ числа

$$
g_{k}=\sum_{i=1}^{\infty} \frac{1}{\lambda_{i}^{k}} .
$$

Справедлива следующая теорема.

Теорема 1. Для любого $\varepsilon>0$, любого натурального $k$ найдется натуральное $n$, зависящее от $k$, что

$$
\left|\sum_{i=1}^{n} b_{i i}^{(k)}(n)-g_{k}\right| \leq \varepsilon .
$$

Здесь $b_{i i}^{(k)}(n)$ - элементы главной диагонали к-й степени матрицы $\mathfrak{R}_{n}(A)$. Кроме того, если $\frac{1}{\left|\lambda_{N}\right|}>\frac{1}{\left|\lambda_{N+1}\right|}$, то для каждого $k \in\{1,2, \ldots, N\}$

$$
\left|\mu_{k}^{(n)}-\frac{1}{\lambda_{k}}\right| \leq \varepsilon
$$

при достаточно больших $n, n \gg N$. Здесь $\mu_{s}^{(n)}$ - собственные числа матрицы $\mathfrak{R}_{n}(A), s=1,2, \ldots, n$, занумерованные по убыванию модулей с учетом алгебраической кратности.

Доказательство. По [1, теорема Лидского] равны матричный и спектральный следы оператора $R_{0}(A)$ (матрицы $\left.\mathfrak{R}_{\infty}(A)\right)$ :

$$
\sum_{i=1}^{\infty} b_{i i}=g_{1} .
$$


Тогда для любого $\varepsilon_{1}>0$ найдется натуральное $N_{1}$ такое, что

$$
\left|\sum_{i=N_{1}+1}^{\infty} b_{i i}\right| \leq \frac{\varepsilon_{1}}{2}
$$

По той же теореме равны матричный и спектральный следы к-й степени оператора $R_{0}(A)$ (матрицы $\left.\mathfrak{R}_{\infty}(A)\right)$ :

$$
\sum_{i=1}^{\infty} b_{i i}^{(k)}=g_{k}
$$

Тогда для любого $\varepsilon_{k}>0$ найдется натуральное $N_{k}$ такое, что

$$
\left|\sum_{i=N_{k}+1}^{\infty} b_{i i}^{(k)}\right| \leq \frac{\varepsilon_{k}}{2}
$$

Другими словами, для любого $k$ можно подобрать такое $N_{k}$, что

$$
\left|\sum_{i=1}^{N_{k}} b_{i i}^{(k)}-g_{k}\right| \leq \frac{\varepsilon_{k}}{2}
$$

Однако, для каждого $k$ можно найти такое большое $n=n(k)$, что

$$
\left|\sum_{i=1}^{n} b_{i i}^{(k)}(n)-\sum_{i=1}^{N_{k}} b_{i i}^{(k)}\right| \leq \frac{\varepsilon_{k}}{2}
$$

поэтому

$$
\left|\sum_{i=1}^{n} b_{i i}^{(k)}(n)-g_{k}\right| \leq\left|\sum_{i=1}^{n} b_{i i}^{(k)}(n)-\sum_{i=1}^{N_{k}} b_{i i}^{(k)}\right|+\left|\sum_{i=1}^{N_{k}} b_{i i}^{(k)}-g_{k}\right| \leq \frac{\varepsilon_{k}}{2}+\frac{\varepsilon_{k}}{2}=\varepsilon_{k} .
$$

Полагая $\varepsilon_{k}=\varepsilon$ для произвольного фиксированного $k$, завершаем доказательство неравенства (1).

Перейдем к доказательству неравенства (2). Пусть натуральное $N$ такое, что $\frac{1}{\left|\lambda_{N}\right|}>\frac{1}{\left|\lambda_{N+1}\right|}$, а $\left\{s_{i}\right\}_{i=1}^{\infty}-$ набор собственных чисел оператора $\sqrt{\left(A^{-1}\right)^{*} A^{-1}}$. Свойства чисел $s_{i}$ таковы, что

$$
s_{i} \geq 0, \quad s_{i} \geq \frac{1}{\left|\lambda_{i}\right|}, \quad \psi_{k}(t) \geq \sum_{i=t+1}^{\infty} \frac{1}{\left|\lambda_{i}\right|^{k}},
$$




$$
\psi_{k}(t)=\sum_{i=t+1}^{\infty} s_{i}^{k}, \quad k \in \mathbb{N}, \quad t \in \mathbb{N} \cup\{0\} .
$$

По [2, теорема 1.1] первые $N$ компонент $z_{k}^{(n)}$ решения системы

$$
\sum_{i=1}^{n} z_{i}^{m}=g_{m}, \quad m=1,2, \ldots, n, \quad n \gg N,
$$

удовлетворяют оценке $\left|z_{k}^{(n)}-w_{k}\right| \leq r_{n, k}, w_{k}=\lambda_{k}^{-1}$, где $r_{n, k}=\left(C \psi_{1}(n)\right)^{1 / \nu_{k}} \rightarrow$ 0 при $n \rightarrow \infty$. Здесь $\nu_{k}-$ алгебраическая кратность собственного числа $\lambda_{k}$, а константа

$$
C=2\left|w_{1}\right|^{\nu_{k}-1} e^{3 \psi_{1}(0) /\left|w_{N+1}\right|}\left(\psi_{1}(0) / \alpha_{N}\right)^{2 \psi_{1}(0) /\left|w_{N}\right|},
$$

где

$$
\alpha_{N}=\min \left\{\left|w_{k}-w_{j}\right|:\left|w_{k}\right| \geq\left|w_{N}\right| / 2,\left|w_{j}\right| \geq\left|w_{N}\right| / 2, w_{k} \neq w_{j}\right\} .
$$

Видим, что константа $C$ зависит лишь от расположения собственных чисел $w_{i}$ ядерного оператора $A^{-1}$ и спектрального следа первого порядка $\psi_{1}(0)>0$ оператора $\sqrt{\left(A^{-1}\right)^{*} A^{-1}}$, причем увеличение следа $\psi_{1}(0)$ на любое ограниченное $\delta>0$ ведет к увеличению на некоторое ограниченное число константы $C$. Поэтому, полагая $S_{m}(n)=\sum_{i=1}^{n} b_{i i}^{(k)}(n)$, по [2, теорема 1.1] первые $N$ компонент $\mu_{k}^{(n)}$ решения новой системы (в то же время $\mu_{k}^{(n)}$ - собственные числа матрицы $\left.\mathfrak{R}_{n}(A)\right)$

$$
\sum_{i=1}^{n} z_{i}^{m}=S_{m}(n), \quad m=1,2, \ldots, n,
$$

будут удовлетворять оценке $\left|\mu_{k}^{(n)}-w_{k}\right| \leq \widetilde{r}_{n, k}$, где

$$
\begin{gathered}
\widetilde{r}_{n, k}=\left(\widetilde{C}\left(\psi_{1}(n)+\varepsilon(n)\right)\right)^{1 / \nu_{k}}, \\
\widetilde{C}=2\left|w_{1}\right|^{\nu_{k}-1} e^{3\left(\psi_{1}(0)+\widetilde{\varepsilon}\right) /\left|w_{N+1}\right|}\left(\left(\psi_{1}(0)+\widetilde{\varepsilon}\right) / \alpha_{N}\right)^{2\left(\psi_{1}(0)+\widetilde{\varepsilon}\right) /\left|w_{N}\right|}, \\
\widetilde{\varepsilon}=\max _{n>N} \varepsilon(n), \quad\left|\sum_{i=1}^{n} \widetilde{s}_{i}^{(n)}-\psi_{1}(0)\right|=\varepsilon(n) .
\end{gathered}
$$

Квадраты чисел $\widetilde{s}_{i}^{(n)} \geq 0$ являются собственными числами матрицы $\left(\mathfrak{R}_{n}(A)\right)^{*} \mathfrak{R}_{n}(A)$, где $\left(\mathfrak{R}_{n}(A)\right)^{*}$ - матрица, эрмитово сопряженная к $\mathfrak{R}_{n}(A)$ : 
$\left(\mathfrak{R}_{n}(A)\right)^{*}=\left(\overline{\mathfrak{R}_{n}(A)}\right)^{t}$. Очевидно, что $\varepsilon(n) \rightarrow 0$ при $n \rightarrow \infty$, поэтому и $\widetilde{r}_{n, k} \rightarrow 0$ при $n \rightarrow \infty$.

Таким образом видим, что для достаточно больших $n$ неравенство (2) выполняется. Теорема доказана.

\section{2. Примеры вычисления.}

Найдем первые собственные числа дифференциальных операторов, описанных в [2, примеры 2 и 3], но уже используя метод Галеркина. Матрица, приближающая резольвенту, будет получена с помощью первых $n$ функций ортонормированного базиса из $\mathbb{H}$.

Пример 1. На отрезке $[0,1]$ задано ДУ второго порядка:

$$
-x y^{\prime \prime}-y^{\prime}=\lambda y
$$

со спектральным параметром $\lambda$ при следующих условиях:

$$
y(x) \text { ограничено при } x \rightarrow 0, y(1)=\alpha y^{\prime}(1), \alpha<0 .
$$

Дифференциальный оператор $-x d^{2} / d x^{2}-d / d x$ с краевыми условиями (4) будем рассматривать как оператор, действующий в $L_{2}(0,1)$.

Выберем ОНБ $\left\{y_{i}(x)\right\} \subset L_{2}(0,1)$, составленный из ортополиномов Лежандра $P_{i}(t), t=2 x-1$, деленных на их нормы. Функция Грина задачи (3)-(4) имеет следующий вид:

$$
G(x, \xi)=G_{1}(x, \xi)= \begin{cases}-\alpha-\ln \xi & \text { при } x \leq \xi \\ -\alpha-\ln x & \text { при } \xi \leq x .\end{cases}
$$

Далее строим матричный оператор

$$
\begin{gathered}
\mathfrak{R}_{n}(A)=\left(b_{i j}\right)_{i, j=1}^{n}, \quad b_{i j}=\left(R_{0}(A) y_{i}, y_{j}\right), \\
R_{0}(A) \varphi(t)=\int_{a}^{b} G(t, s) \varphi(s) d s
\end{gathered}
$$

и пусть количество первых функций из ОНБ будет $n=10$. Вычисляя собственные числа симметрической матрицы методом вращения Якоби или методом бисекции, выделим из них три наибольшие по модулю:

$$
\begin{aligned}
& \mu_{1}^{(10)}=2.5364733279074727311, \\
& \mu_{2}^{(10)}=0.24035372772217389528, \\
& \mu_{3}^{(10)}=0.078116666801071284655, \\
& \text { поэтому }
\end{aligned}
$$




$$
\begin{aligned}
& \lambda_{1} \approx 1 / \mu_{1}^{(10)}=0.39424818270215168122, \\
& \lambda_{2} \approx 1 / \mu_{2}^{(10)}=4.1605345982231035791, \\
& \lambda_{3} \approx 1 / \mu_{3}^{(10)}=12.801365456958873898 .
\end{aligned}
$$

Результаты той же задачи, но уже методом следов степеней резольвент (см.[2, пример 2]):

$z_{1}^{(10)}=2.5364733279074727295$,

$z_{2}^{(10)}=0.24035373403229174197$,

$z_{3}^{(10)}=0.077911068320642988917$,

откуда

$\lambda_{1} \approx 1 / z_{1}^{(10)}=0.39424818270215168146$,

$\lambda_{2} \approx 1 / z_{2}^{(10)}=4.1605344889946627325$,

$\lambda_{3} \approx 1 / z_{3}^{(10)}=12.835146809751089072$.

Пример 2. Пусть на отрезке [0,1] задана следующая спектральная задача:

$$
y^{I V}=\lambda y
$$

со спектральным параметром $\lambda$ при следующих условиях:

$$
y(0)=y^{\prime}(0)=y(1)=y^{\prime}(1)=0 .
$$

Дифференциальный оператор $d^{4} / d x^{4}$ с краевыми условиями (6) пусть действует в пространстве $L_{2}(0,1)$.

ОНБ $\left\{y_{i}(x)\right\} \subset L_{2}(0,1)$ составлен из ортополиномов Лежандра $P_{i}(t)$, $t=2 x-1$, деленных на их нормы, а функция Грина задачи (5)-(6):

$$
G(x, \xi)= \begin{cases}\left(\frac{\xi}{2}-\xi^{2}+\frac{\xi^{3}}{2}\right) x^{2}-\left(\frac{1}{6}-\frac{\xi^{2}}{2}+\frac{\xi^{3}}{3}\right) x^{3}, \quad x \leq \xi, \\ \left(\frac{x}{2}-x^{2}+\frac{x^{3}}{2}\right) \xi^{2}-\left(\frac{1}{6}-\frac{x^{2}}{2}+\frac{x^{3}}{3}\right) \xi^{3}, \quad \xi \leq x .\end{cases}
$$

Строим матричный оператор $\mathfrak{R}_{n}(A)=\left(b_{i j}\right)_{i, j=1}^{n}$ и, также как в предыдущей задаче, пусть количество первых функций из ОНБ будет $n=10$. Собственные числа симметрической матрицы могут быть вычислены любыми методами, в частности, методом вращения Якоби или методом бисекции. Наибольшие по модулю три собственных числа:

$\mu_{1}^{(10)}=0.0019977469340468297122$
$\mu_{2}^{(10)}=0.00026291317167458561327$ 
$\mu_{3}^{(10)}=0.000068408002419678096201$, поэтому

$\lambda_{1} \approx 1 / \mu_{1}^{(10)}=500.56390174220071487$,

$\lambda_{2} \approx 1 / \mu_{2}^{(10)}=3803.5370903277744093$,

$\lambda_{3} \approx 1 / \mu_{3}^{(10)}=14618.172795999407658$.

Методом следов степеней резольвент наибольшие по модулю три собственных числа вычислены так (см.[2, пример 3]):

$z_{1}^{(10)}=0.0019977469340538862620$,

$z_{2}^{(10)}=0.00026291317235406293856$,

$z_{3}^{(10)}=0.000068410541891813517397$,

отсюда

$\lambda_{1} \approx 1 / z_{1}^{(10)}=500.56390174043259598$,

$\lambda_{2} \approx 1 / z_{2}^{(10)}=3803.5370804978477243$,

$\lambda_{3} \approx 1 / z_{3}^{(10)}=14617.630153864736086$.

Видим, результаты обоих методов очень схожи.

\section{Список литературы}

[1] Садовничий В. А. Теория операторов / В. А. Садовничий. - изд. 2 - М. : Изд-во Моск. ун-та, 1986. - 386 с.

[2] Малеко Е. М. О методе следов резольвент, вычисленных точно / Е. М. Малеко // Вестник СамГУ - Естественнонаучная серия. 2011. № 5(86). - 220 c. 